\title{
...meanwhile, elsewhere in Italy
}

SIR - Success in sports, high-school performance and scientific creativity seem to correlate with birth dates ${ }^{1-4}$. Recent observations by our group suggest that genetic and developmental parameters in newborn infants bear a relationship with season of birth.

The search for a possible adaptative the population of Penne, a small rural town 438 metres above sea level in southeast Italy. The majority of the people admitted to the Town Hospital (including puerperae) live between 400 and 500 metres above sea level, but there are villages located at higher altitude. Cold winters characterize the climate of this area.

\begin{tabular}{lcccc}
\hline \multicolumn{5}{c}{ ACP $_{1}^{*}$ A GENE IN A SAMPLE OF NEWBORN FROM THE POPULATION OF PENNE } \\
\hline Month of birth & $\begin{array}{c}\text { ACP }{ }^{*} \text { A } \\
\text { (\%) }\end{array}$ & $\begin{array}{c}\text { Gestation duration } \\
\text { (weeks) }\end{array}$ & $\begin{array}{c}\text { Birth weight } \\
\text { (g) }\end{array}$ & $\begin{array}{c}\text { Sex proportion } \\
(\%)\end{array}$ \\
Oct./Nov. & $35.9 \pm 3.7$ & $39.4 \pm 0.13$ & $3,272 \pm 56$ & $51.8 \pm 5.4$ \\
Dec./Jan. & $35.5 \pm 3.5$ & $39.5 \pm 0.15$ & $3,345 \pm 51$ & $51.6 \pm 5.2$ \\
Feb./Mar. & $25.0 \pm 3.2$ & $39.7 \pm 0.16$ & $3,429 \pm 46$ & $45.7 \pm 5.2$ \\
Apr./May & $31.6 \pm 4.7$ & $40.1 \pm 0.07$ & $3,423 \pm 64$ & $47.9 \pm 7.2$ \\
Jun./Sept. & $19.7 \pm 4.9$ & $39.6 \pm 0.21$ & $3,383 \pm 61$ & $45.6 \pm 8.7$ \\
\hline
\end{tabular}

value of $\mathrm{ACP}$ (acid phosphatase controlled by locus 1) was the main purpose of our survey. The enzyme is a phosphotyrosine phosphatase and may have important functions in cellular growth regulation and in the modulation of glycolytic rate ${ }^{5}$. Population studies suggest a role in adaptation to environmental temperature ${ }^{6}$ and we have recently shown associations with obesity and diabetes $^{7,8}$.

We studied 352 newborn infants from
The table shows $\mathrm{ACP}^{*} \mathrm{~A}$ frequency, gestational length, birth weight and sex of newborn infants from the population of Penne. ACP *A, the allele associated with the lowest $\mathrm{ACP}$ activity, gestational length and birth weight show a significant association with season of birth $(P<0.01 \%)$. Most seasonal heterogeneity is due to differences between infants born in October-January and infants born in February-September, corresponding to conception in January-April and May-December respectively. Infants born from October to January show a higher frequency of $\mathrm{ACP}_{1}{ }^{*} \mathrm{~A}$, a reduced gestational duration and a lower birth weight compared with infants born from February to September. This pattern is in line with population and biochemical studies suggesting that low-activity genotypes may have an increased metabolic rate and a relative advantage in cold environments ${ }^{6}$.

Our observations suggest that season of conception and/or environmental temperature may influence intrauterine development. The effects on neonatal parameters measurable at a general population level seem rather small, but may be far-reaching in selected groups of talented people.

\section{F. Gloria-Bottini \\ N. Lucarini}

M. La Torre

Department of Preventive Pediatrics, University of Rome Tor Vergata,

School of Medicine,

o0173 Rome, Italy

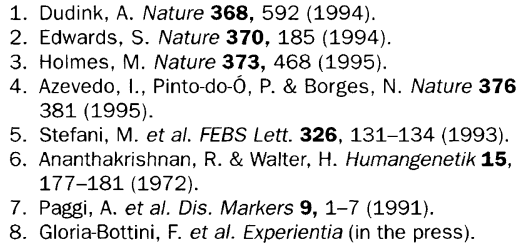

\section{Automatically determine HLA types with tools from an $\AA \mathrm{A} O \mathrm{O}$ country?}

Can you say your method of determining HLA types is completely accurate? If not, a new approach to detecting HLA types offers you real accuracy.

Introducing HLA SBTyper, ${ }^{\mathrm{TM}}$ the new software for automatic sequence based typing from Pharmacia Biotech in Sweden-an $\AA \ddot{A ̈ O ̈ ~ c o u n t r y . ~}$

HLA SBTyper uses raw sequence data, generated by ALFexpress, ${ }^{\mathrm{TM}}$ to automatically generate high-resolution HLA typing for class II genes. It determines HLA types by comparing sequence results against a database of well defined and established subtypes. And it all takes just two minutes. ALFexpress and HLA SBTyper also let you access raw data-so you can always go back and verify your results.

HLA SBTyper with AutoLoad ${ }^{\text {TM }}$ Solid Phase Sequencing Kit and ALFexpress-together they're the tools that present you with the most accurate way of determining HLA types, and handle every step from capture of PCR products to evaluation.

Just call us at 1 (800) 5263593 in the United States or +4618165011 from the rest of the world. Ask for more information about what we Swedes have done for automated sequence based HLA typing. You'll find we've taken every å (pronounced ooh) ä (aah) and ö (uh) out of determining HLA types. 